\title{
EFEITO DE REGULADORES DE CRESCIMENTO SOBRE OS TEORES DE ÁCIDO ASCÓRBICO E CARBOIDRATOS SOLÚVEIS DE MORANGO (Fragaria hybridus)*
}

\author{
Luiz Eduardo Gutierrez ** \\ Paulo R. C. Castro *** \\ Keigo Minami **** \\ Wilson P. Cesar Junior $* * * * *$
}

\section{RESUMO}

Foram feitas aplicações dos seguintes reguladores de crescimento em morango (Fragaria hybridus): SADH a $5000 \mathrm{ppm}$, CCC a $2000 \mathrm{ppm}$, ATA a $10 \mathrm{ppm}$ (3 aplicações), ácido giberélico a $10 \mathrm{ppm}$ (3 aplicaçôes) e $550 \mathrm{ppm}$. Análises de peso seco, ácido ascórbico e carboidratos solúveis dos frutos foram efetuadas a fim de se estudar o efeito desses reguladores de crescimento. Não foram detectadas diferenças significativas entre os diversos tratamentos e o controle. O peso seco variou de 7,62 a 9,53\%. O conteúdo de ácido ascórbico variou de 35,88 a $71,81 \mathrm{mg} / 100 \mathrm{~g}$ de peso fresco. Os teores médios de carboidratos solúveis, expresso em $\mathrm{g} / 100 \mathrm{~g}$ peso fresco, foram: totais $(5,58)$, sacarose $(1,01)$, glucose $(1,63)$ e frutose $(1,54)$.

\section{INTRODUÇÃO}

Reguladores de crescimento têm sido aplicados em morangos visando, entre outros fatores, maior número de flores, tamanho e número de frutos (PATHAKI \& SINGH, 1971; CELESTRE \& PIERANDREI, 1971). Entretanto, poucas são as referências sobre os efeitos na qualidade dos frutos. No presente trabalho, diversos reguladores de crescimento em diferentes doses foram aplicados em moranguinho a fim de se estudar o efeito sobre os teores de ácido ascórbico e carboidratas solúveis .

* Entregue para publicação em 20-10-77.

** Departamento de Química, E.S.A. "Luiz de Queiroz".

*** Departamento de Botânica, E.S.A. "Luiz de Queiroz".

**** Departamento de Agricultura e Horticultura, ESALQ.

***** Estagiário junto ao Departamento de Química. 
Comparando-se dados citados por diversos autores, verifica-se uma variação nos teores de ácido ascórbico de morangos. Segundo EZELL et alii (1947) e ROBINSON (1949), a síntese de ácido ascórbico em morango é estimulada por radiações luminosas, sendo o mesmo fato observado por GURGEL et alii (1951) para goiaba.

Outros fatores para justificar a variação nos teores de ácido ascórbico estariam nas variedades utilizadas (SLATE \& ROBINSON, 1946); OVERCASH \& McWHIRTER, 1948), na aplicação de herbicidas no solo (ZAVARZIN \& BELJAEVA, 1967; ANPALOV et alii, 1969), no efeito de radiações gama (CHACHIN et alii, 1969), da época de colheita (LUNDERGAN \& MOORE, 1957a), maturidade dos frutos (EZELL et alii, 1947) e tamanho dos frutos (SLATE \& ROBINSON, 1946) .

STANCHEN (1974) verificou que a aplicação de $\mathrm{MnCl}_{2}$ provocou aumento no acúmulo de sacarose e ácido ascórbico no fruto enquanto que $\mathrm{ZnSO}_{4}$ não apresentou efeito significativo.

Não foram encontradas correlações entre a coloração e o teor do ácido ascórbico dos frutos (LUNDERGAN \& MOORE, 1975b) e que as condições ótimas para a formação da coloração não foram as mais desejáveis para ácido ascórbico (LUNDERGAN \& MOORE, 1975a) .

GOMES (1973) citou que levulose é o principal açúcar encontrado no morango, enquanto que CHOUREITAH \& BÚNEMANN (1970) revelaram a presença de glucose, frutose, sacarose, xilose a maltose. Os açúcares livres constituem cerca de 70 a $80 \%$ dos sólidos solúveis totais nos frutos maduros (CULPEPPER et alii, 1935) enquanto MONEY \& CHRISTIAN (1950) revelaram níveis de $50 \%$.

Os herbicidas aplicados no solo provocaram profundas alterações nos teores de sacarose, glucose e frutose, ocorrendo redução nos açúcares totais (ZAVARZIN \& BELJAEVA, 1967).

Os teores de matéria seca de morango aumentaram com o amadurecimento e foram maiores nos frutos da segunda colheita (STOJKOVSKA \& SIMOVSKI, 1969) e em geral variam de 5 a $10 \%$ (CULPEPPER et alii, 1935; OVERCASH \& McWHIRTER, 1948; MONEY \& CHRISTIAN, 1950; DUEWER \& ZYCH, 1967 e GOMES, 1973).

\section{MATERIAL E MÉTODOS}

O experimento foi realizado no Campo Experimental do Setor de Horticultura da ESALQ, Piracicaba (SP), iniciado em 24 de abril de 1975 . 
Foram utilizadas plantas de Fragaria Hybridus var. Monte Alegre, distribuídas no espaçamento de $40 \times 40 \mathrm{~cm}$, com coberturas com serragens de madeira, tendo recebido a seguinte adubação: $50 \mathrm{~g} /$ cova de 5-10-10 e $10 \mathrm{~g} /$ cova de sulfato de amônio com cobertura aos 20, 40 e 60 dias do transplante. A colheita dos frutos iniciou-se em 8-07-75 e a última em 12-09-75.

Os tratamentos utilizados foram os seguintes:

\begin{tabular}{|c|c|}
\hline Tratamentos & Reguladores de crescimento \\
\hline 1 & Controle \\
\hline 2 & SADH $5000 \mathrm{ppm}$ \\
\hline 3 & CCD $2000 \mathrm{ppm}$ \\
\hline 4 & IAA $\quad 10 \mathrm{ppm}$ (3 aplicações) \\
\hline 5 & GA $\quad 10 \mathrm{ppm}$ (3 aplicações) \\
\hline 6 & GA $\quad 550 \mathrm{ppm}$ \\
\hline
\end{tabular}

Para análise estatística foi realizado um delineamento de blocos ao acaso com 5 repetições, adotando-se o nível de 5\% de probabilidade para comparação ds médias pelos testes F e Tukey (PIMENTEL GOMES, 1970).

As amostras foram obtidas na fase de maior produção e para análise cerca de 20 frutos maduros foram homogeneizados.

A matéria seca foi obtida por secagem em estufa a 100-1059C até peso constante.

Os teores de ácido ascórbico foram obtidos pelo método colorimétrico de 2,6 diclorofenol-indofenol descrito em JACOBS (1958) .

A extração de carboidratos solúveis foi realizada com água fervente por 30 minutos após homogeinização em VIRTZ durante 2 minutos.

A determinação de carboidratos solúveis totais foi realizada segundo método descrito por DUBOIS et alii (1956) utilizando glucose como padrão.

A separação e identificação de açúcares foi feita por cromotografia em papel de filtro segundo técnica de ARZOLLA \& FONSECA (1965) . A quantificação foi realizada pelo método de DUBOIS et alii (1956) . 


\section{RESULTADOS E DISCUSS ÃO}

Os dados apresentados para matéria seca citados no QUADRO 1, estão de acordo com observações feitas por CULPEPPER et alii (1935), OVERCASH \& MCWHIRTER (1948), MONEY \& CHRISTIAN (1950), DUEWER \& ZYCH (1967) e GOMES (1973) . A matéria seca dos frutos não foi afetada significativamente pela ação dos reguladores de crescimento, variando de 7,62 a $9,53 \%$.

Não foi detectado efeito significativo dos reguladores de crescimento, nas doses utilizadas, sobre os teores de ácido ascórbico de morangos (QUADRO 2), que variaram de 35,88 a $71,81 \mathrm{mg} / 100 \mathrm{~g}$ polpa fresca. Fato este em desacordo com TAVADZE \& MAZANASHVILI (1972) que observaram incremento no conteúdo de ácido ascórbico de frutos tratados com ácido giberélico.

Os teores de ácido ascórbico apresentados no QUADRO 2 estão de acordo com os dados apresentados por SLATE \& ROBINSON (1946), EZELL et alii (1947), OVERCASH \& McWHIRTER (1948), ROBINSON (1949) e LUNDERGAN \& MOORE (1975b). Entretanto, foram ligeiramente inferiores aos citados por FONSECA et alii (1969) e superiores aos apresentados SHAULIS (1956).

Os QUADROS de 3 a 6 apresentam os teores de carboidratos solúveis totais, glucose, frutose e sacarose, respectivamente. Não foram detectadas diferenças, significativas entre os tartamentos e o controle, indicando provavelmente nenhum efeito dos reguladores de crescimento, nas doses utilizadas, sobre carboidratos solúveis de morango, embora TAVADZE \& MAZANASHVILI (1972) tenham relatado que a aplicação de ácido giberélico aumentou o teor de açúcares nos frutos.

Os teores de carboidratos solúveis totais apresentados no QUADRO 3 estão de acordo com os dados citados por CULPEPPER et alii (1935), MONEY \& CHRISTIAN (1950) e GOMES (1973) .

Os valores médios de carboidratos solúveis apresentados no QUADRO 7 mostram a predominância de açúcares redutores (glucose e frutose) na fração carboidratos apresentaram uma relação aproximada de glucose/frutose/sacarose de 1,5/1,5/1,0.

A relação açúcares simples/carboidratos totais foi maior nos frutos que receberam reguladores de crescimento em comparação com o controle.

Apenas glucose, frutose e sacarose foram detectadas nos extratos aquosos de morangos confirmando observações de ZAVARZIN \& BELJAEVA (1967) enquanto que CHOUREITAH \& BƯNEMANN (1970) detectaram também a presença de xilose e maltose. 
A fração carboidratos solúveis totais contribuiu com cerca de $67 \%$ do peso seco dos frutos, sendo que CULPEPPER et alii (1935) citou uma variação de 70 a $80 \%$. Os açúcares livres detectados no presente trabalho foram responsáveis por aproximadamente $50 \%$ da matéria seca.

Não foi encontrada correlação significativa entre a matéria seca e carboidratos solúveis totais embora este constituinte contribua com $70 \%$ do peso seco. Também não foi encontrada correlação significativa entre os teores de sacarose e açúcares redutores (glucose + frutose).

QUADRO 1 - Efeito de reguladores de crescimento sobre matéria seca de morangos.

\begin{tabular}{|c|c|c|c|c|c|c|}
\hline \multirow[b]{2}{*}{ Tratamentos (*) } & \multicolumn{6}{|c|}{ Repetições } \\
\hline & $\mathrm{A}$ & $B$ & $\mathrm{C}$ & $\mathrm{D}$ & $\mathrm{E}$ & Médias \\
\hline 1 & 8,04 & 8,08 & 8,86 & 8,04 & 8,31 & 8,27 \\
\hline 2 & 7,77 & 8,50 & 7,62 & 7,78 & 8,04 & 7,94 \\
\hline 3 & 9,53 & 8,29 & 8,51 & 8,05 & 8,02 & 8,48 \\
\hline 4 & 8,16 & 7,99 & 8,56 & 8,87 & 8,12 & 8,34 \\
\hline 5 & 9,21 & 8,09 & 8,63 & 7,98 & 8,48 & 8,48 \\
\hline 6 & 9,32 & 7,70 & 9,05 & $|9,37| * *$ & 8,20 & 8,53 \\
\hline
\end{tabular}

(*) Consultar a Secção Material e Métodos

C. V. $=6,29 \% \quad$ Teste F não foi significativo

$\left.\right|^{* *} \mid=$ Estimado estatisticamente.

QUADRO 2 - Efeito de reguladores de crescimento sobre o teor de ácido ascórbico de monangos (Expressos em $\mathrm{mg} / 100 \mathrm{~g}$ polpa iresca).

Repetiçots

\begin{tabular}{|c|c|c|c|c|c|c|}
\hline Tratamentos (*) & $\mathrm{A}$ & B & $\mathrm{C}$ & $\mathrm{D}$ & $\mathrm{E}$ & Médias \\
\hline 1 & 60,23 & 35,88 & 54,44 & 54,51 & 55,13 & 52,04 \\
\hline 2 & 41,68 & 57,99 & 53,99 & 58,63 & 46,34 & 51,73 \\
\hline 3 & 56,70 & $60^{\prime} 20$ & 58,45 & 57,82 & 71,81 & 61,00 \\
\hline 4 & 55,96 & 60,66 & 53,38 & 38,80 & 58,49 & 53,46 \\
\hline 5 & 56,15 & 57,32 & 63,39 & 37,80 & 45,98 & 52,13 \\
\hline 6 & 47,37 & 53,26 & 46,58 & $|+8,69| * *$ & 70,35 & $5,3,25$ \\
\hline
\end{tabular}

(*) Consultar a Secção Material e Métodos

$|* *|$ Estimado estatisticamente $\quad$ C.V. $=16,96 \%$

Teste $F$ não foi significativo 
QUADRO 3 - Efeito de reguladores ae crescimento sobre o teor de carboidratos solúteis totais de morangos (Expresso em $\mathrm{g} / 100 \mathrm{~g}$ de polpa fresca).

\begin{tabular}{ccccccc}
\hline & \multicolumn{7}{c}{ Repetiçóes } \\
\cline { 2 - 6 } Tratamentos $\left(^{*}\right)$ & A & B & C & D & E & Médias \\
\hline \hline 1 & 5,23 & 5,67 & 4,84 & 5,73 & 5,78 & 5,45 \\
2 & 6,22 & 5,45 & 5,58 & 5,30 & 5,63 & 5,64 \\
3 & 5,37 & 5,80 & 5,61 & 5,50 & 5,12 & 5,48 \\
6 & 5,75 & 5,82 & 4,83 & 6,38 & 5,78 & 5,71 \\
4 & 6,45 & 5,97 & 5,39 & 5,18 & 5,73 & 5,74 \\
5 & 4,74 & 5,69 & 6,24 & $|5,56| * *$ & 5,11 & 5,47 \\
\hline
\end{tabular}

(*) Consultar a Secção Material e Métodros

|** Estimado estatisticamente $\quad$ C.V. $=8,83 \%$

Teste $F$ não foi significativo

QUADRO 4 - Efeito de reguladores de crescimento sobre o teor de glucose de morangos (Expresso em $\mathrm{g} / 100$ de polpa fresca).

\begin{tabular}{ccccccc}
\hline & \multicolumn{7}{c}{ Repetiçôes } \\
\cline { 2 - 6 } Tratamentos $\left(^{*}\right)$ & $\mathrm{A}$ & $\mathrm{B}$ & $\mathrm{C}$ & $\mathrm{D}$ & $\mathrm{E}$ & Médias \\
\hline \hline & & & & & \\
\hline & 1,69 & 1,48 & 1,28 & 1,62 & 1,35 & 1,48 \\
3 & 1,86 & 1,72 & 1,51 & 1,69 & 1,76 & 1,71 \\
4 & 1,56 & 1,45 & 1,81 & 1,63 & 1,67 & 1,62 \\
5 & 1,84 & 1,92 & 1,32 & 1,88 & 1,75 & 1,74 \\
6 & 1,94 & 1,49 & 1,54 & 1,52 & 1,48 & 1,59 \\
& 1,81 & 1,40 & 1,65 & $|1,68| * *$ & 1,69 & 1,65 \\
\hline
\end{tabular}

(*) Consultar a Secção Material e Métodos

$\left.\right|^{* *} \mid$ Fstimado estatisticamente $\quad$ C.V. $=9,87 \%$

Teste $F$ não foi significativo 
QUADRO 5 - Efeito de reguladores de crescimento sobre o teor de frutose de morangos (Expresso em $\mathrm{g} / 100$ de polpa fresca).

\begin{tabular}{|c|c|c|c|c|c|c|}
\hline \multirow[b]{2}{*}{ Tratamentos (*) } & \multicolumn{6}{|c|}{ Repetições } \\
\hline & A & $\mathrm{B}$ & $\mathrm{C}$ & $\mathrm{D}$ & $\mathrm{E}$ & Médias \\
\hline 1 & 1,32 & 1,15 & 1,28 & 1,51 & 1,27 & 1,31 \\
\hline 2 & 2,03 & 1,87 & 1,37 & 1,61 & 1,55 & 1,69 \\
\hline 3 & 1,63 & 1,68 & 1,91 & 1,38 & 1,44 & 1,61 \\
\hline 4 & 1,57 & 1,74 & 1,45 & 1,67 & 1,80 & 1,65 \\
\hline 5 & 1,70 & 1,31 & 1,36 & 1,61 & 1,68 & 1,53 \\
\hline 6 & 1,30 & 1,43 & 1,67 & $|1,47|^{* *}$ & 1,50 & 1,47 \\
\hline
\end{tabular}

(*) Consultar a Secção Material e Métodos

$\left.\right|^{* *} \mid$ Estimado estatisticamente C.V. $=13,16 \%$

Teste $\mathbf{F}$ não foi significativo

QUADRO 6 - Efeito de reguladores de crescimento sobre o teor de sacarose de morangos (Expresso em g/100 de polpa fresca).

\begin{tabular}{|c|c|c|c|c|c|c|}
\hline \multirow[b]{2}{*}{ Tratamentos $(*)$} & \multicolumn{6}{|c|}{ Repetições } \\
\hline & A & $\mathrm{B}$ & $\mathrm{C}$ & $\mathrm{D}$ & $\mathrm{E}$ & Médias \\
\hline 1 & 0,84 & 0,93 & 1,10 & 0,90 & 0,85 & 0,92 \\
\hline 2 & 1,18 & 0,86 & 1,22 & 0,83 & 0,96 & 1,01 \\
\hline 3 & 1,05 & 0,79 & 1,12 & 1,16 & 1,21 & 1,07 \\
\hline 4 & 1,31 & 0,84 & 0,65 & 0,86 & 0,81 & 0,89 \\
\hline 5 & 1,20 & 1,07 & 0,92 & 1,09 & 1,05 & 1,07 \\
\hline 6 & 0,97 & 0,88 & 1,13 & $|1,06| * *$ & 1,39 & 1,09 \\
\hline
\end{tabular}

(*) Consultar a Secção Material e Métodos

$|* *|$ Estimado estatisticamente C.V. $=17,44 \%$

Teste $F$ não foi significativo 
QUADRO 7 - Carboidratos solúveis de morango (médias de 5 repetições, expressos em $\mathrm{g} / 100 \mathrm{~g}$ de polpa fresca).

\begin{tabular}{|c|c|c|c|c|c|c|}
\hline \multirow[b]{2}{*}{ Carboidratos solúveis } & \multicolumn{6}{|c|}{ Tratamentos (*) } \\
\hline & 1 & 2 & 3 & 4 & 5 & 6 \\
\hline Totais & 5,45 & 5,64 & 5,48 & 5,71 & 5,74 & 5,47 \\
\hline Glucose & 1,48 & 1,71 & 1,62 & 1,74 & 1,59 & 1,65 \\
\hline Frutose & 1,31 & 1,69 & 1,61 & 1,65 & 1,53 & 1,47 \\
\hline Sacarose & 0,92 & 1,01 & 1,07 & 0,89 & 1,07 & 1,09 \\
\hline Açúcares simples/totais & 0,68 & 0,78 & 0,78 & 0,75 & 0,73 & 0,77 \\
\hline
\end{tabular}

(*) Consultar a Secção Material e Métodos

\section{CONCLUSÕES}

Com os resultados obtidos, as seguintes conclusões podem ser apresentadas:

1. Os reguladores de crescimento nas doses utilizadas não apresentaram efeitos significativos sobre os teores de matéria seca, ácido ascórbico e carboidratos solúveis de morango.

2. Carboidratos solúveis totais constituíram cerca de $67 \%$ do peso seco dos frutos, enquanto que glucose, frutose e sacarose representaram $50 \%$ do peso seco.

3. Os frutos de morango apresentaram predominância de açúcares redutores (glucose e frutose).

\section{SUMMARY}

"THE EFFECT OF GROWTH REGULATORS ON ASCORBIC ACID AND SOLUBRE CARBOHYDRATES CONTENTS OF STRAWBERRY FRUIT

(FRAGARIA HYBRIDUS)"

Several growth regulators were sprayed on strawberry plants: SADH (5000 ppm), CCC (2000 ppm), IAA (10 ppm, 3 times), GA $10 \mathrm{ppm}, 3$ times) and GA (550 ppm), Ascorbic acid, dry weight and soluble carbohydrates contents of fruits were determined. 
Statistically differences were not observed between treatments and control. Dry weight varied from 7.62 to $9.53 \%$. Ascorbic acid content varied from 35.88 to $71.81 \mathrm{mg} / 100 \mathrm{~g}$ on fresh weight basis. Mean values of soluble carbohydrates, in grams $/ 100 \mathrm{~g}$ on fresh weight basis, were total (5.58), sucrose (1.01), glucose (1.63) and frutose (1.54).

\section{LITERATURA CITADA}

ANPALOV, V.A., V.A. ZAKORDONEC and O.A. DROZDOVA. 1969. Herbicides and the quality of strawberries. Zasc. Rast. 14: 25-26. In: Horticultural Abstracts, 10: 705 .

ARZOLLA, J.D.P. \& H. FONSECA. 1965. Cromatografia de Açúcares. Boletim Didático n. ${ }^{\circ}$ 7. E.S.A. "Luiz de Queiroz". $19 \mathrm{p}$.

CELESTRE, M.R. and PIERANDREI. 1971. The effect of gibberellic acid on productivity and ripening date in three strawberry varieties. Annali dell'Instituto Sperimentale per la Frutticoltura, 2:61-76. In: Horticultural Abstracts, 43:358.

CHACHIN, K., K. KATO and K. OGATA. 1969. Effects of gama radiation on decay, some chemical compositions and quality of strawberry. J. Jap. Soc. Hort. Sci., 38: 101-118. In: Horticultural Abstracts, 40:88.

CHOUREITAH, A. and G. BUNEMANN. 1970. The effect of the $\mathrm{K}$ supply on the constituents of strawberries. Gartenbaunwissenschaft, 35:419--427. In: Horticultural Abstrats, $41: 741$.

CULPEPPER, C.W., J.S. CALDWELL and H.H. MOON. 1935. A physiological study of development and ripening in the strawberry. J. Agric. Res., 50:645-696.

DUBOIS, M., K.A. GILlES, J.K. HAMILTON, P.A. REBERS and F. SMITH. 1956. Colorimétric method for determination of sugars and related substances. Anal. Chem., $28: 350-356$.

DUEWER, R.G. and C.C. ZYCH. 1967. Heritability of soluble solids and acids in progenies of the cultivated strawberry (Fragaria v. Ananalssa Duch.). Proc. Amer. Soc. Hort. Sci., $90: 153-157$.

EZELL, B.D., G.M. DARROW, M.S. WILCOX and D.H. SCOTT. 1947. Ascorbic acid content of strawberries. Food Res., 12:510-526.

FONSECA, H., J.N. NOGUEIRA e A.M.S. MARCONDES. 1969. Teor de ácido ascórbico e beta-caroteno em frutas e hortaliças brasileiras. Archivos Latinoamericanos de Nutrición, 19:9-16.

GOMES, P. 1973. Fruticultura Brasileira. Livraria Nobel. São Paulo. 446 p.

GURGEL, J.T.A., J. SOUBIHE SOBRINHO, E. MALAVOLTA e J. LEME JUNIOR. 1951. Fatores que afetam a determinação de vitamina $C$ na goiaba (Psidium guajava L.) Anais da E.S.A. "Luiz de Queiroz", 8: 399-432.

JACOBS, M.B. 1958. The Chemical Analysis of Foods and Foods Products. Van Nostrand. New York. 971 p. 
LUNDERGAN, C.A. and J.N. MOORE. 1975a. Variability in vitamin C content and colour of strawberries in Arkansas. Arkansas Farm Rescarch, 24:2. In: Horticultural Abstracts, $46: 183$.

LUNDERGAN, C.A. and J.N. MOORE. 1975b. ' Inheritance of ascorbic acid content and color intensity in fruits of strawberry (Fragaria $\mathrm{x}$ ananassa Luch.). J. Ainer. Soc. Hort. Sci., $100: 633-635$.

MONEY, R.W. and W.A. CHRISTIAN. 1950. Analytical data of some common fruits. J. Sci. Food Agric., 1:8-12.

OVERCASH, J.P. and L. McWHIRTER. 1948. The ascorbic acid content of nine strawberry varieties in Mississipi. Proc. Amerc. Soc. Hort. Sci., 52: 251-256.

PATHAK, R.K. and R. SINGH. 1971. Effect of some external factors on the growth and fruiting of strawberry. II. Effect of G.A., growth retardants and cheching on flowering and yield. Progressive Horticulture, 3:53-63. In: Horticultural Abstracts, $44: 86$.

PIMENTEL GOMES, F. 1970. Curso de Estatistica Experimental. 4. ${ }^{\mathrm{a}}$ ed. Livraria Nobel. São Paulo. 430 p.

ROBINSON, W.B. 1949. The effect of sunlight on the ascorbic acid content of strawberry. J. Agric. Res., 78: 257-262.

SHAULIS, N. 1956. The sampling of small fruits for composition and nutritional studies. Proc. Amer. Soc. Hort. Sci., $68: 576-586$.

SLATE, G.L. and W.B. ROBINiSON. 1946. Ascorbic acid content of strawberry varieties and selections at Geneva, New York in 1945. Proc. Amer. Soc. Hort. Sci., $47: 219-223$.

STANCHEV, L.B. 1974. Effect of manganese and zinc on the quality of strawberries. Pochnoznanie i Agrokhimiya, 9:62-67. In: Horticultural Abstracts, $45: 505$.

STOJKOVSKA, A. and K. SIMOVSKI. 1969. The contents of dry matter, organic acids and ascorbic acid in some strawberries varieties in the Skopje region. Annu. Fac. Agric. Sylvic. Skopje Agric., $22: 85-93$. In: Horticultural Abstracts, $41: 103$.

TAVADZE, P.G. and T.G. MAZANASHVILI. 1972. The effect of gibberellic acid on the growth and yield of large-fruited strawberries. Academiya Nauk. Gruz. SSR, 5 : 13-15. In: Horticultural Abstracts, $44: 198$.

ZAVARZIN, V.I. and T.V. BELJAEVA. 1967. The effect of herbicides on the yield and quality of strawberries. Himija sel'. Hoz., 5:35-37. In: Horticultural Abstracts, $38: 661$. 